Corresponding Author:

Teresa Arora; email:

Teresa.Arora@zu.ac.ae

Production and Hosting by Knowledge E

(c) Teresa Arora et al. This article is distributed under the terms of the Creative

commons Attribution

License, which permits unrestricted use and redistribution provided that the original author and source are credited.

Editor-in-Chief:

Dr. Dimitrios Papandreou

Official Publication of Zayed University, UAE

\title{
The Role of Genetic, Dietary and Lifestyle Factors in Pediatric Metabolic Syndrome: A Review of the Literature from Prenatal to Adolescence
}

Teresa Arora ${ }^{1,2}$, Sahar Agouba², Ahmad Sharara², and Shahrad Taheri²

1Zayed University, Department of Psychology, Abu Dhabi, United Arab Emirates

${ }^{2}$ Weill Cornell Medicine in Qatar, Clinical Research Core, Doha, Qatar

\section{Abstract}

The metabolic syndrome (MetS) is described as a cluster of health conditions that are associated with an increased risk of cardiovascular disease. The clinical diagnosis of MetS in pediatrics is challenging due to differing criteria, although the estimated prevalence continues to rise. The increased prevalence of childhood obesity and insulin resistance, in both developed and developing countries, is believed to be a major contributor to MetS diagnosis in children. We review the current literature surrounding genetic predisposition, maternal influence, epigenetics, environmental and lifestyle factors pertaining to pediatric MetS with a specific emphasis on obesity and insulin resistance. We highlight and discuss recent, key studies in prenatal through to adolescent populations and review evidence suggesting that children may be pre-disposed to obesity and insulin resistance, prenatally. We also discuss several key lifestyle drivers of these conditions including poor nutrition and dietary habits, insufficient physical activity, use of electronic devices, over-consumption of caffeinated and/or sugar-sweetened beverages, as well as the importance of sleep during childhood and adolescence in relation to metabolic health. We conclude with recommendations for preventable methods to tackle this growing pediatric public health issue, which, if current trends continue, will undoubtedly compromise the health and longevity of the next adult generation.

Keywords: metabolic syndrome, obesity, pediatrics, diet, physical activity

\section{Introduction}

The metabolic syndrome is a constellation of cardio-metabolic abnormalities that were observed to cluster and increase cardiovascular risk. Several hypotheses have been proposed suggesting common etiological mechanisms for the metabolic syndrome revolving around central adiposity and insulin resistance. The components of the 
metabolic syndrome depend on the definitions used by various international bodies. Generally, components include: 1) elevated triglycerides; 2 ) low levels of high density lipoprotein-cholesterol (HDL-C); 3) hypertension; 4) glucose intolerance/insulin resistance; and 5) excess adiposity (usually determined through waist circumference or body mass index (BMI) cut points). If an individual has three or more components, they are designated to have the metabolic syndrome.

The metabolic syndrome is usually observed in older populations, but with an increasing prevalence of obesity in younger populations, it is becoming more common in younger individuals with potentially serious downstream repercussions for health. The definition of the metabolic syndrome in younger populations has not reached consensus and different definitions exist. With central adiposity playing a central role in the metabolic syndrome, the syndrome has a strong genetic link, influenced by key environmental factors that promote excess nutrition and physical inactivity. Two components of the metabolic syndrome, type 2 diabetes and obesity, are increasingly observed in children and adolescents $[36,65]$ and these are believed to drive the onset of MetS [21, 32]. Indeed, prospective data has shown that excess adiposity and metabolic abnormalities in pediatric populations can result in adverse cardiometabolic profiles in adulthood $[49,81]$. The Bogalusa Heart Study showed that childhood obesity tracks into adulthood [29]. Furthermore, longitudinal evidence has shown that the presence of overweight/obesity and/or metabolic abnormality at age 924 years predicts an increased risk of metabolic syndrome, type 2 diabetes mellitus and adverse cardiovascular outcomes 21-25 years later [47]. The problem of excess adiposity with attendant insulin resistance in adolescents is now resulting in highly invasive bariatric surgery procedures [24]. Despite these surgeries demonstrating a significant reduction in MetS prevalence from $27 \%$ to $2 \%$ [50], this treatment strategy remains controversial. Alternative treatments include targeting lifestyle behavior modification. However, success of these types of interventions can only occur once a better understanding of all contributory factors, and the extent to which they are involved, has been determined. The focus of this review is to highlight and discuss the most recent evidence surrounding factors that contribute to the onset and progression of two closely related, and increasingly prevalent, diseases in childhood (obesity and insulin resistance), which are major drivers of the global epidemic of pediatric Mets.

\section{Literature Search}

We searched PubMed database using the following search terms: 'metabolic syndrome' AND 'children' 'adolescent' 'pediatric'. Filters for age (o-18 years), human 
research and English language were applied and we restricted the search to highlight articles published in the last five years (2011-2016). Our search revealed a total of 420 articles, which were subsequently examined based on relevance to the current review. Further, we also reviewed and included relevant articles from reference sections of identified manuscripts as well as other known literature deemed pertinent to the review.

\section{The Contribution of Genetics and Epigenetics upon the Risk of Obesity}

It was recently purported that $10 \%$ of obesity cases can be explained by genetics and that $90 \%$ are attributable to environmental factors (discussed in later sections) [78]. Whilst the embryo develops in the intrauterine environment, multiple maternal genetic and lifestyle factors can predispose the fetus to later obesity [74]. Furthermore, interactions between the environment and genes following birth, has been linked to Deoxyribonucleic Acid (DNA) methylation alterations [74]. For example, monozygotic twins are identical, epigenetically, in the early stages of life. Progressively, however, alterations to the genetic distribution of 5 -metylcytosine DNA have been noted as well as changes in histone acetylation [74]. This suggests that exposure to differing environmental stimuli may result in distinguishable genetic features. MicroRNAs are involved in the regulation of epigenetics. The influence of nutrition upon epigenetics has been previously highlighted involving methyl-group metabolism. During important developmental periods, ingestion of foods containing choline, methionine and folate, can alter DNA and histone methylation [80]. This results in chronic alterations to gene expression and the epigenetics that may predispose children to obesity later in life [80].

Oxidative stress is common in the pathogenesis of metabolic disorders and may contribute to the development of cardiovascular disease and type 2 diabetes. However, the precise role of antioxidant enzymes in the prevention of metabolic diseases is not completely understood. A recent study examined the role of Paraoxonase 1 (PON1) polymorphism (Q192R) in relation to insulin resistance in 117 children (6-12 years old) [2]. Q192R genotypes were characterized and the genotype of each sample was determined, generating three allelic clusters: QQ, QR and RR. Insulin resistance was derived using the homeostasis model assessment (HOMA-IR). An association between the polymorphism in those with the RR genotype and insulin resistance ( $\geq 95^{\text {th }}$ percentile) (odds ratio $[O R]=4.55 ; 95 \%$ confidence intervals $[\mathrm{Cl}]: 1.21-18.53$ ) was observed. An increased risk was shown for RR carriers compared to other genotypes (OR $=6.38$, P 
$<0.01)$ [2]. Other recent genetic studies have highlighted the potential influence of polymorphisms upon metabolic health in pediatric populations $[53,77]$. Despite growing evidence in the field of genetics and its link to hormone regulation, the importance of maternal health as a contributor to MetS in children has received much attention.

\section{The Importance of Maternal Health in Relation to the Growth, Development and Metabolic Profile of the Offspring}

Maternal health has been highlighted as a key factor for the health and development of offspring in the intrauterine environment [26]. A recent study, which examined data from 937 women and their offspring, examined the prospective relationships between fasting glucose levels during pregnancy upon multiple outcomes of the offspring's development from birth to 3 years [4]. A positive relationship was observed between gestational fasting glucose level and birth weight $(\beta=0.17, \mathrm{p}<0.001)$. Obesity in mothers before pregnancy, although self-reported, was positively associated with conditional growth in standardized BMI of the infant between birth and 1-year ( $\beta=$ $0.10, \mathrm{P}=0.018)$, birth to 18 months $(\beta=0.11, \mathrm{P}=0.024)$, birth to 2 years $(\beta=0.14$, $\mathrm{p}=0.002)$ as well as at 3 years $(\beta=0.19, \mathrm{p}<0.001)$, suggesting a dose-response association [4]. This study highlights the importance of maternal health in relation to subsequent growth and development of the offspring. Gestational diabetes, even when less severe, has been linked to larger birth weights and large-for-gestational age (LGA) when compared to mothers with normal glucose tolerance [43].

Further support for the importance of maternal health, even prior to conception, relating to subsequent cardiometabolic risk in the offspring has been shown. Project Viva, which recruited 1,090 mother-child pairs, demonstrated that each 5-unit increase in maternal BMI pre-pregnancy was associated with a $0.92 \mathrm{~kg}$ increase in total fat and $0.39 \mathrm{~kg}$ trunk fat in the offspring at birth. Each $5 \mathrm{~kg}$ of weight gained during pregnancy was positively and significantly associated with greater total and trunk adiposity (determined using dual $\mathrm{x}$-ray absorptiometry) in the offspring, after adjustment [64]. Whilst mothers with gestational diabetes and pre-pregnancy obesity are show to be more likely to deliver offspring that are LGA, the negative consequences associated with LGA have been shown to persist well into childhood [30]. The longterm consequences of children born LGA include imbalances in glucose homeostasis with higher insulin resistance pre-pubertally compared to normal for gestational age counterparts [30]. 
Clearly, maternal health, both before and during pregnancy, has important implications for the future metabolic profile of the offspring. The mother's role in the health and development of their offspring is undisputable, including decisions about feeding methods from birth. Recent evidence has supported a link between breastfeeding and the future cardiometabolic health of the offspring. The large prospective study of 727 children, followed from birth until four years of age examined the impact of three different methods of feeding at three months as well as breastfeeding duration. Compared to children exclusively or predominantly breastfed at three months, those who were not breastfed had significantly higher mean BMI, and higher total cholesterol levels. Furthermore, children breastfed for less than three months compared to those breastfed for more than 12 months had a 0.44 increase in mean BMI at age four years [66]. This study highlights the long-term benefits of breastfeeding upon the future metabolic health of children, although there are several considerations. Breastfeeding is usually obtained from maternal reports and could be subject to social desirability bias. The use of objective measures for determining excess adiposity and adipose distribution in relation to feeding methods during infancy in relation to childhood obesity is preferable. Future studies in this area now require longer follow up given that the mean increase of $0.44 \mathrm{BMI}$ over a four-year period is unlikely to be clinically relevant.

Although these studies demonstrate the importance of maternal health and early decisions about feeding during infancy that may predispose children to insulin resistance and obesity, exposure to the external environment and negative health behaviours during childhood and adolescence provide additional clues about factors that support the progression of chronic conditions associated with MetS.

\section{The Contribution of Negative Health Behaviours in Relation to Obesity and Insulin Resistance in Children and Adolescents}

Repeated exposure to negative health behaviours observed in childhood has been consistently linked to the subsequent practice of unhealthy lifestyle behaviours. A clear example of this is the contribution of parental obesity when investigating childhood obesity, given that this is one of the strongest predictors [57]. At a translational level, negative health habits of parents are commonly mirrored in their offspring. We will now review and discuss the contribution of a series of behaviours that are key for optimal metabolic health and body weight homeostasis in children and adolescents. 


\subsection{Sleep}

The importance of achieving a balance between sleep and wakefulness was noted at least four decades ago in the Alameda County Study [11]. The study emphasized seven social and psychological aspects in relation to health and longevity, one of which was sleep duration [11]. This advice is not restricted to adults but also applicable to children and adolescents, with different age groups requiring different sleep quantities. More recently, sleep and has been recognized as a supplementary lifestyle factor, important for health and longevity $[8,9]$. Sleep is crucial in childhood and contributes to healthy physical and neural development, yet sleep problems are common in pediatrics. The impact of insufficient sleep or poor quality sleep in pediatric populations has been extensively examined with findings consistently reporting associations between sub-optimal sleep features and increased BMI as well as insulin resistance and type 2 diabetes mellitus [40] [3, 42, 55, 73] [6]. A recent study showed that sleeping 7-9 hours had a protective effect on MetS in adolescent $(\mathrm{OR}=0.38$, 95\% Cl: 0.15-0.94) [25]. Mechanistic explanations for the association between sleep and metabolic abnormalities have been linked to alterations in metabolic hormones related to hunger and appetite. Not meeting individual sleep requirements can influence energy balance and may result in behaviours that promote obesity such as physical inactivity and unhealthy food intake (discussed in later sections).

The focus of attention between sleep and metabolic dysfunction has shifted with more attention now concentrating on sleep architecture. Sleep architecture (sleep staging) in relation to glucose metabolism has been explored in children and adolescents, with an initial focus on those with obesity $[28,46]$. A recent study investigated the effect of sleep staging upon glucose tolerance, insulin sensitivity and pancreatic $\beta$ cell function in children and adolescents ( $n=118$; mean age 13.1 years; $45 \%$ boys) [82]. Sleep outcomes were assessed using the gold standard measure (polysomnography) for one-night and a 2-hour oral glucose tolerance test was administered on the subsequent morning. The amount of time spent in slow wave sleep (SWS) was significantly and positively associated with insulin sensitivity. Conversely, the amount of time spent in light sleep (stage 1 ) was negatively associated with insulin sensitivity. The study findings found further support for a positive linear association between sleep duration and glucose tolerance [82].

The first study to examine the effect of experimental sleep manipulation upon insulin resistance in lean adolescents was conducted by Klingenberg and colleagues [45]. This randomized crossover study assessed 21 healthy males (mean age 16.8 years), subjecting them to three nights of either short (four hours time in bed) or 
long (nine hours time in bed) sleep opportunity. Insulin resistance was determined from pre and post-prandial levels of glucose and insulin at the end of each sleep condition. Whilst they observed no change in glucose level between the two sleep conditions, insulin resistance significantly increased by $65 \%$ following sleep restriction compared to the nine-hour sleep opportunity [45]. Interestingly, adolescents with insulin resistance compared to those without, have been shown to have a reduced amount of stage 2 and 3 sleep (light and deep sleep, respectively). Deep sleep, also known as slow wave sleep, is known for its restorative properties including cell repair. Thus, a reduced amount of slow wave sleep activity is likely to produce downstream effects on hormone secretion and metabolic function. Notably, sleepdisordered breathing (SDB) is associated with disruptions to sleep architecture and has become increasingly recognized in pediatric populations to OSA diagnosis in children). Interestingly, more severe obstructive sleep apena (OSA), a SDB condition, has also been linked to greater insulin resistance and elevated glucose levels in children [70]. Given previous reports of a close relationship between OSA and MetS in adolescents [68] and the increasing prevalence of these conditions in pediatric populations, SDB is an important consideration for future research studies in this area.

There are many features of sleep to be considered. For example, sleep timing and circadian rhythmicity are essential sleep parameters to scrutinize. Delays in circadian sleep-wake patterns are common during adolescence due to a combination of pubertal transition and psychosocial factors. A recent study examined the effect of circadian preference upon BMI in a large sample $(n=511$ ) of young adolescents (aged 11 -13 years) and found that those with a later preference had significantly greater BMI z-scores ( $\beta$ $=0.51, \mathrm{p}<0.01$ [ [8]. Furthermore, the study also revealed those with later preferences had poorer dietary habits [8]; thus alterations to sleep and circadian imbalance have the potential to disrupt energy homeostasis and well as appetite regulating hormones [38], which in turn, may result in weight gain and insulin resistance.

\subsection{Diet}

The importance of a healthy diet and optimum nutrition has been consistently emphasized in recent decades through national campaigns following recognition of poor dietary habits. This has been highlighted as a result of exposure to an 'obesogenic' environment. Rapid changes and increased exposure to food availability, including processed, ready-made and fast foods, has been purported to influence dietary intake. These modified foods tend to have higher sugar, salt and calorie contents. Over-consumption of these food items is likely to result in positive energy, which 
in turn, promotes obesity and/or insulin resistance if levels of energy expenditure are insufficient to counteract energy imbalance. Campaigns have aimed at educating individuals to improve dietary behaviours have included information about healthy portion sizes, reducing sugary food/beverage intake and increasing healthy food selection (consumption of five portions of fruit/vegetables per day). Despite raised awareness and dietary guidelines, recent prospective evidence from a birth cohort in Australia revealed that, on average, less than half of the total sample met the requirements at either 14 or 17 years of age [17]. Moreover, further support for a significant negative linear association between meeting dietary guidelines and insulin resistance was reported [17]. The most recent evidence surrounding the contribution of diet in relation to MetS in children has focused on examining diet types (vegetarian and Mediterranean), specific foods (white rice) and macronutrients (saturated fat), each discussed in turn.

The beneficial effects of a Mediterranean diet upon cardiovascular health have been extensively reported in adults $[14,34,35,48,67,79]$ although less evidence is available in pediatric populations. In particular, the effect of a Mediterranean-style diet in children at high risk of MetS, has been recently explored, revealing positive results [76]. Children and adolescents ( $n=49$; mean age 11 years) attending a family medical center were included if they had $B M I \geq 95^{\text {th }}$ percentile as well as any other feature of MetS, according to the International Diabetes Federation (IDF) criteria [1]. Of the total sample, 24 were randomized to receive education and instructions to follow a Mediterranean-style diet and the remaining 25 followed a standard diet for 16 weeks, supported by parents [76]. Anthropometric measures (height, weight, waist and circumference, and bioelectrical impedance), blood pressure and fasting blood samples were recorded/obtained at baseline and at the end of the 16-week dietary intervention. Food recall (24-hour) was used to quantify calorie, macro and micronutrient content every three weeks throughout the intervention period. Those in the Mediterranean diet group had a significant mean reduction in BMI, fat mass, lean mass, as well as glucose, total cholesterol, low-density lipoprotein (LDL) and triglyceride levels at the end of the intervention compared to baseline. An increase in levels of high-density lipoprotein (HDL) was also observed in the same group. Furthermore, the number of MetS components significantly decreased from baseline to the end of the 16-week intervention in those following the Mediterranean diet and meeting the criteria for MetS decreased by $45 \%$ [76]. Whilst these study results provide powerful support for promoting a Mediterranean diet, caution with interpretation of the findings is necessary. Limitations of the study include 1) small sample size; 2) individualized recommended daily calorie intake based on age and sex-specific cut points rather 
than basal metabolic rate; 3) relatively short intervention period with no report on compliance to the diet condition at each of the three weekly assessments; 4 ) between group differences were not reported, which is important given that the standard diet group also received lifestyle and dietary advice with suggested modification; 5) the differences between the two diet interventions were minimal; 6) no assessment of continued compliance to the diet or MetS monitoring beyond the study period; 7) no adjustment was performed for any potential confounders; and 8) subjective dietary measures, possibly resulting in social desirability and recall bias.

The Mediterranean diet is largely plant-based, with the exception of fish. A recent review of the literature suggests that a vegetarian diet can reduce all-cause and specific-cause mortality (cancer, cardiovascular, type 2 diabetes mellitus) [69]. The beneficial effect of fruit and vegetable consumption in childhood has been prospectively assessed in relation to components of MetS by at least two groups. A large cohort ( $n=2,218$ ) of children and adolescents (aged 3-18 years at baseline), followed for $\sim 27$ years found a $14 \%$ decreased risk of developing MetS in those with a higher intake of vegetables during childhood. Furthermore, the study also revealed that higher vegetable consumption in childhood decreased the risk of high blood pressure (OR = 0.88, 95\% Cl: $0.80-0.98$ ) and elevated triglycerides (OR $=0.88,95 \% \mathrm{Cl}: 0.79-0.99)$ at the time measured during adulthood [41]. The Child-Adolescent Blood Pressure Study reported similar findings following assessment of food intake derived from a 106item food frequency questionnaire administered to 1,764 children and adolescents (aged 6-19 years) [56]. A Registered Dietitian condensed food items into seven categories: grains, nuts, fruits, vegetables, dairy, meats and low-nutrient-dense foods. Those in the highest quartile of grains, vegetables, nuts and low-nutrient-dense foods consumption had a reduced risk of being overweight (BMI $>85^{\text {th }}$ percentile), where corresponding ORs (95\% Cls) were 0.59 (0.41-0.83), 0.67 (0.48-0.94), 0.60 (0.43$0.85)$ and $0.43(0.29-0.63)$, respectively. Whilst the categories of nuts and vegetable consumption are consistent with other study findings $[41,62]$, the most surprising finding was that of low-nutrient-dense foods which was shown as a protective factor for overweight. The authors explained this by suggesting that consumption of foods in this category may have been under-reported, possibly due to social acceptance [56], which may be a common issue when assessing dietary habits.

A balanced diet involves the incorporation of multiple macronutrients. Carbohydrate content and glycemic load have been recent focuses in nutritional research. Efforts have been made to examine the effect of white rice intake in adolescents upon MetS and its components, in populations where rice is a staple food. Data obtained from the Fourth Korea National Health and Nutrition Examination Survey, during 2007-2009, 
was used [72]. Information was available from 2,209 adolescents (aged 10-18 years) and features of MetS were objectively acquired. Diet was assessed using 24-hour food recall and white rice consumption was divided into quartiles. Analysis was performed according to gender. Girls in the highest quartile of white rice consumption had greater insulin resistance $(p=0.005)$ and lower HDL-cholesterol $(p<0.001)$, compared to the other three quartiles. Girls in the two upper quartiles of white rice intake were more likely to meet the criteria for MetS $(p=0.003)$ [72]. These findings were not found in boys and explanation for this is possibly related to differences in insulin-like growth factors and/or sex hormones during pubertal transition. However, gender differences have been observed in multiple adult studies $[23,59,60]$ suggesting that women with high white rice/carbohydrate/glycemic load have more adverse metabolic profiles, compared to men. The authors purported that high consumption of white rice may result in altered glucose metabolism and dyslipidemia [72]. The macronutrient breakdown and proportion of each category (fats, carbohydrates, protein) is clearly important and has been the recent focus of a randomized controlled trial.

Nupponen and colleagues report on findings from the Special Turku Coronary Risk Factor Intervention Project (STRIP) [61]. The trial aimed to reduce children's intake of saturated fat through dietary counseling and to examine the effectiveness of the program upon preventing later atherosclerosis. The study recruited five-month old infants and parents (1990-1992). At six months of age, 1,062 infants and their parents were randomly assigned to either the intervention $(n=540$ ) or control condition ( $n$ $=522$ ). The intervention group received individualized dietary counseling every two years until the infant reached 20 years of age. The primary aim of the intervention was to replaced saturated fat with unsaturated fat in the diet of the child, which was supported by counseling and nutritional advice. Counseling was parent-focused until the child was 7 years old. Beyond this age, the advice gradually increased to be more child-focused. Food records were used to make suggestions about methods for improving diet. The control group was also seen every two years and received basic health education as part of standard Finnish care. Individual components of MetS were measured at study visits when the child was 15-20 years old. As previously described, there is no consensus on universal criteria for MetS in pediatric populations therefore the study applied five separate definitions. This resulted in a range (6.0$7.5 \%$ ) for meeting a diagnosis of MetS in the intervention group and $10-14 \%$ in the control group between age 15-20 years. The long-term risk of developing MetS in the intervention group was significantly lower (relative risk [RR] = 0.59, 95\% Cl: 0.40-0.88) compared to the control group. There was further evidence to support the efficacy of the intervention for individual components of MetS including a decreased risk of 
hypertension where $\mathrm{RR}=0.8395 \% \mathrm{Cl}: 0.70-0.99$ and high triglycerides in males $(\mathrm{RR}=$ $0.71,95 \% \mathrm{Cl}$ : 0.52-0.98). The authors concluded that repeated dietary advice, targeted at reducing saturated fat intake during childhood, reduces the risk of MetS and its associated features [61].

Undoubtedly, diet is a crucial lifestyle component for ensuring a healthy metabolic profile later on in life. Many studies that examine the relationships between lifestyle factors believed to predict MetS and its features, do not adjust for the effect of diet, which is a major concern and should be addressed in future studies. Parental involvement and education concerning diet, and other contributory yet modifiable behaviours, during early parenthood and beyond may be necessary for optimizing the future health and longevity of the offspring.

\subsection{Screen Time}

Over the last decade, there has been a surge in the availability and accessibility of electronic devices. Portable equivalents of what used to be static media (television, telephones, computers and gaming consoles) are now increasingly available and ownership has increased amongst children and adolescents. Engaging in the use of these devices has been purported to promote unhealthy lifestyles by increasing levels of sedentariness (see next section). Other consequences include limitation of face-toface interactions, resulting in social isolation/loneliness, lowered self-confidence and addiction [12], which has been prospectively linked to circadian disruption in pediatrics [18]. Children, from a young age, may be permitted and encouraged to use electronic media communication devices with tablet holders now available to secure onto pushchairs. Whilst there are many benefits of these devices, concerns about the impact upon health have been raised. Early data from the National Health And Nutrition Examination Survey (NHANES) revealed a dose-dependent association between the amount of daily screen time (television and computer use) and MetS in 12-19 year olds [54]. After adjustment for physical activity level, the risk of MetS was more than three-fold for adolescents spending five hours per day on screens compared to those using screens for one-hour or less [54]. These findings are consistent with more recent evidence from the same survey, conducted in Korean children and adolescents. Compared to the lowest quartile of screen time use ( $\leq 16$ hours per week), those in the highest quartile ( $\geq 35$ hours per week) were more than twice as likely to meet the criteria for MetS after adjustment (OR = $2.2395 \%$ Cl: 1.02-4.86) [44].

Progressive technology use with increasing age in adolescents has been associated with increased levels of sedentariness/physical inactivity [20,37], possibly displacing 
activities involving greater energy expenditure. Persistent use can therefore result in obesity development. In combination, obesity onset may be exacerbated through over-consumption of foods that are high in calories, fat and sugar. Some have shown that exposure to advertisements marketing unhealthy foods trigger intake of these food items [27, 31], which in turn, can promote weight gain and subsequent obesity. Paradoxically, preliminary evidence has shown that video games involving physical activity can enhance weight loss in overweight/obese children [19, 52, 75]. The relationship between technology use and obesity and/or insulin resistance is, however, convoluted.

The complex interactions between technology use and childhood obesity/metabolic dysfunction may be mediated by sleep [7], food/beverage intake [20] and physical inactivity [20]. In a study of 738 young adolescent (11-13 years old), the use of six different types of technology at bedtime was examined in relation to eight sleep features [5]. More frequent use of all technology before bedtime was associated with multiple adverse effects on sleep [5]. Explanations for technology use resulting in adverse sleep outcomes are mental excitation/stimulation and exposure to blue light, emitted from electronic devices, which can suppress melatonin release and delay sleep onset. The same group examined the mediating effect of sleep duration upon the established relationship between technology use and adolescent BMI [7]. Bedtime use of all four technologies assessed was related to shorter sleep duration [7]. A negative association was also observed between sleep duration and BMI ( $\beta=-0.40, \mathrm{P}$ $<0.01$ ), consistent with other studies. Sleep duration was recognized as a mediator of the relationship between portable types of technology (mobile telephones and computers) and BMI [7]. This suggests the direct association between screen-time and obesity in children/adolescents is complex, requiring consideration of multiple factors and lifestyle behaviours.

The use of technology in teenagers is particularly problematic given that this group commonly experience circadian phase delay during pubertal transition. This results in later bed times with more free time in the evening, which may be consumed by engaging with devices. Recent evidence has shown that $94 \%$ of adolescent boys and $87 \%$ of girls spent more than 2 hours, daily, engaging in screen-based activities [20]. A concomitant behavior is the consumption of caffeinated beverages and/or sugarsweetened beverages in adolescents $[15,20,23]$. Both of these behaviors, whether examined singularly or in combination, have been linked to poorer sleep outcomes 
and/or obesity $[5-7,16,51]$, and are also likely to influence metabolic regulation. Consideration should be given to this emerging area of study, particularly as both technology use and consumption of sugar-dense beverages with low nutritional content, have been shown to increase with age in pediatric populations [37, 51].

Most recent evidence from a large prospective cohort of children, suggests that interventions aimed at reducing screen time and sedentariness in children at risk of obesity, may help to alleviate the risk of MetS development [39]. This recommendation reinforces the notion that modification to multiple lifestyle factors, which are known drivers of MetS and its individual components, need to be tackled in a timely manner, one of which is sedentariness.

\subsection{Sedentariness}

Low levels of physical activity reflect sedentariness and long, persistent episodes can result in positive energy and subsequent obesity and/or insulin resistance, particularly when combined with other negative related health behaviours. The contribution of energy expenditure upon metabolic health is well recognized. One of the largest studies to date to examine the association between levels of sedentariness and central obesity was conducted in 124,113 Greek school-aged children ( $9.9 \pm 1.1$ years old, 51\% male). A seven-day recall questionnaire was administered which asked about diet and physical activity habits and anthropometric measures were obtained [33]. Central obesity was prevalent in $33.4 \%$ of the sample and revealed significant associations between the condition and frequent (at least four times per week) breakfast consumption (OR $=0.7295 \% \mathrm{Cl}$ : 0.69-0.75), habitual snack consumption both at midday and the afternoon ( $\mathrm{OR}=0.7095 \% \mathrm{Cl}: 0.67-0.74)$ and high levels of sedentariness, defined as participation in sedentary activities $\geq 4$ times per week, OR $=1.10$ (95\% Cl: 1.07-1.14). The authors concluded that emphasis should be placed on regular meal consumption and reducing levels of sedentariness [33].

A recent cross-sectional study examined the contribution of diet and physical activity levels upon the metabolic profiles of 667 Chilean adolescents (16-17 years old; $52.2 \%$ male) [13]. Of the total sample $9.5 \%$ met the criteria for MetS. Physical activity was measured using a validated, standardized questionnaire with scores ranging 0-10. Physical inactivity was determined using a cut point of $\leq 3$ and was recognized as a major contributor to MetS, where $\mathrm{OR}=2.9$ (95\% Cl: 1.1-7.7) [13]. Whilst the importance of consistent physical activity in childhood can be emphasized for later metabolic and cardiovascular health, the school environment may inadvertently impede adequate levels of activity. 
Sedentariness is a particular problem in the academic environment [22] where children are required to remain seated throughout the majority of lessons. Findings from a recently published randomized controlled trial (RCT) aiming to address this concern reported positive, albeit minimal, improvements to obesity in children [58]. The study compared findings of the intervention group, where children performed one daily physical activity session of one-hour during school time, to the control group, which undertook two physical activity sessions per week over a four-year timeframe [58]. Mean BMI and proportion of childhood overweight were similar across the two groups at baseline. At the end of the four-year intervention period, a larger proportion of those in the intervention group were between the $10-90^{\text {th }}$ percentile for BMI compared to the control group, $87 \%$ versus $78 \%$, respectively. However, this difference was not statistically significant $(p=0.13)$. Childhood overweight $\left(B M I>90^{\text {th }}\right.$ percentile) was consistently lower in the intervention group compared to the control group across all annual assessments. However, the authors present no formal statistical analysis for these relationships and it therefore remains unclear if these findings were statistically significant or not. The high withdrawal rate of overweight/obese children (44\%) across the total sample is also likely to have influenced the findings [58]. Whilst this is the most recent RCT to report on the effectiveness of overcoming sedentariness in an academic setting, additional studies need to be performed in this area. The importance of an active lifestyle requires educating both children and parents to ensure that the appropriate level of support, attention, application and reinforcement is provided, outside of the academic environment.

\section{Recommendations for Tackling MetS in Pediatrics}

Educational interventions in mothers, before, during, and beyond pregnancy appear to be paramount for reducing the risk of later obesity in the offspring. Parents are major role models to their children and early, persistent exposure to healthy lifestyle practices ensure that these are more likely to be adopted by the offspring. Moreover, modification of parental behaviors influence the child's and has been shown to reflect similar behavioral change [71]. Addressing, and attempting to reverse, negative health-related behaviors at an early age are necessary to encourage healthy lifestyles and minimize the onset of metabolic disorders. Modification of unhealthy lifestyle habits, that are becoming more prevalent in contemporary society (later bedtimes, exposure to electronic media at night-time, consumption of energy/caffeinated drinks, sedentariness and poor diet), should be specifically targeted and minimized wherever possible. Successful interventions are likely to require parental involvement, which 
could help to curb the rising prevalence of childhood obesity, insulin resistance and MetS. Methods to reduce increasing levels of sedentariness in children and adolescents, both in the home and academic environment, are urgently required.

\section{Conclusions}

Maternal health, even prior to conception, plays a crucial role in the growth, development and future metabolic profile of the offspring. The metabolic health of women should be investigated and advice provided to those in the planning stages of pregnancy. Weight loss in those with excess adiposity before conceiving should be targeted. Excessive weight gain during pregnancy should be managed in an attempt to improve the future cardiometabolic outcomes of the offspring. Parental involvement, (particularly the mother) at all stages of an intervention aimed at children, is likely to be fundamental to the success of future trials. Further study of epigenetics and the relevance of specific polymorphisms is also required to better understand the influence the metabolic profile of children and adolescents. Sleep optimization and education about the importance of this behavior, which is currently overlooked, may be a promising tool and contribute to healthy metabolic regulation in pediatric populations. Clearly, a holistic, multi-disciplinary approach targeting multiple health-related behaviors is now required.

\section{References}

[1] Alberti G, Zimmet P, Shaw J, Bloomgarden Z, Kaufman F, Silink M, et al. Type 2 diabetes in the young: the evolving epidemic: the international diabetes federation consensus workshop. Diabetes Care. 2004;27 (7):1798-811.

[2] Alegria-Torres JA, Garcia-Dominguez ML, Cruz M, Aradillas-Garcia C. Q192R polymorphism of paraoxonase 1 gene associated with insulin resistance in Mexican children. Arch Med Res. 2015;46 (1):78-83.

[3] Androutsos O, Moschonis G, Mavrogianni C, Roma-Giannikou E, Chrousos GP, Kanaka-Gantenbein C, et al. Identification of lifestyle patterns, including sleep deprivation, associated with insulin resistance in children: the Healthy Growth Study. Eur J Clin Nutr. 2014;68 (3):344-9.

[4] Aris IM, Soh SE, Tint MT, Saw SM, Rajadurai VS, Godfrey KM, et al. Associations of gestational glycemia and prepregnancy adiposity with offspring growth and adiposity in an Asian population. Am J Clin Nutr. 2015;102 (5):1104-12. 
[5] Arora T, Broglia E, Thomas GN, Taheri S. Associations between specific technologies and adolescent sleep quantity, sleep quality, and parasomnias. Sleep Med. 2014;15 (2):240-7.

[6] Arora T, Hosseini-Araghi M, Bishop J, Yao GL, Thomas GN, Taheri S. The complexity of obesity in U.K. adolescents: relationships with quantity and type of technology, sleep duration and quality, academic performance and aspiration. Pediatr Obes. 2013;8 (5):358-66.

[7] Arora T, Hussain S, Hubert Lam KB, Lily Yao G, Neil Thomas G, Taheri S. Exploring the complex pathways among specific types of technology, self-reported sleep duration and body mass index in UK adolescents. Int J Obes (Lond). 2013;37 (9):125460.

[8] Arora T, Taheri S. Associations among late chronotype, body mass index and dietary behaviors in young adolescents. Int J Obes (Lond). 2015;39 (1):39-44.

[9] Arora T, Taheri S. Sleep Optimization and Diabetes Control: A Review of the Literature. Diabetes Ther. 2015;6 (4):425-68.

[10] Arora T. Sleep Doesn't Waste Time, It's Good for the Waist Line. Sleep. 2015;38 (8):1159-60.

[11] Belloc NB, Breslow L. Relationship of physical health status and health practices. Prev Med. 1972;1 (3):409-21.

[12] Boniel-Nissim M, Lenzi M, Zsiros E, de Matos MG, Gommans R, Harel-Fisch Y, et al. International trends in electronic media communication among 11- to 15-year-olds in 30 countries from 2002 to 2010: association with ease of communication with friends of the opposite sex. Eur J Public Health. 2015;25 Suppl 2:41-5.

[13] Burrows R, Correa-Burrows P, Reyes M, Blanco E, Albala C, Gahagan S. High cardiometabolic risk in healthy Chilean adolescents: associations with anthropometric, biological and lifestyle factors. Public Health Nutr. 2016;19 (3):486-93.

[14] Buscemi S, Nicolucci A, Mattina A, Rosafio G, Massenti FM, Lucisano G, et al. Association of dietary patterns with insulin resistance and clinically silent carotid atherosclerosis in apparently healthy people. Eur J Clin Nutr. 2013;67 (12):1284-90.

[15] Calamaro CJ, Yang K, Ratcliffe S, Chasens ER. Wired at a young age: the effect of caffeine and technology on sleep duration and body mass index in school-aged children. J Pediatr Health Care. 2012;26 (4):276-82.

[16] Centers for Disease C, Prevention. Beverage consumption among high school students - United States, 2010. MMWR Morb Mortal Wkly Rep. 2011;60 (23):77880. 
[17] Chan She Ping-Delfos WL, Beilin LJ, Oddy WH, Burrows S, Mori TA. Use of the Dietary Guideline Index to assess cardiometabolic risk in adolescents. $\mathrm{Br}$ J Nutr. 2015;113 (11):1741-52.

[18] Chen YL, Gau SS. Sleep problems and internet addiction among children and adolescents: a longitudinal study. J Sleep Res. 2016;25 (4):458-65.

[19] Christison A, Khan HA. Exergaming for health: a community-based pediatric weight management program using active video gaming. Clin Pediatr (Phila). 2012;51 $(4): 382-8$.

[20] Christofaro DG, De Andrade SM, Mesas AE, Fernandes RA, Farias Junior JC. Higher screen time is associated with overweight, poor dietary habits and physical inactivity in Brazilian adolescents, mainly among girls. Eur J Sport Sci. 2016;16 (4):498-506.

[21] Cornier MA, Dabelea D, Hernandez TL, Lindstrom RC, Steig AJ, Stob NR, et al. The metabolic syndrome. Endocr Rev. 2008;29 (7):777-822.

[22] De Decker E, De Craemer M, De Bourdeaudhuij I, Wijndaele K, Duvinage K, Androutsos 0 , et al. Influencing factors of sedentary behavior in European preschool settings: an exploration through focus groups with teachers. J Sch Health. 2013;83 (9):654-61.

[23] Demissie Z, Lowry R, Eaton DK, Park S, Kann L. Electronic media and beverage intake among United States high school students-2010. J Nutr Educ Behav. 2013;45 (6):75660.

[24] Dillard BE, 3rd, Gorodner V, Galvani C, Holterman M, Browne A, Gallo A, et al. Initial experience with the adjustable gastric band in morbidly obese US adolescents and recommendations for further investigation. J Pediatr Gastroenterol Nutr. 2007;45 (2):240-6.

[25] Fadzlina AA, Harun F, Nurul Haniza MY, Al Sadat N, Murray L, Cantwell MM, et al. Metabolic syndrome among 13 year old adolescents: prevalence and risk factors. BMC Public Health. 2014;14 Suppl 3:S7.

[26] Faienza MF, Wang DQ, Fruhbeck G, Garruti G, Portincasa P. The dangerous link between childhood and adulthood predictors of obesity and metabolic syndrome. Intern Emerg Med. 2016;11 (2):175-82.

[27] Falbe J, Willett WC, Rosner B, Gortmaker SL, Sonneville KR, Field AE. Longitudinal relations of television, electronic games, and digital versatile discs with changes in diet in adolescents. Am J Clin Nutr. 2014;100 (4):1173-81.

[28] Flint J, Kothare SV, Zihlif M, Suarez E, Adams R, Legido A, et al. Association between inadequate sleep and insulin resistance in obese children. J Pediatr. 2007;150 $(4): 364-9$. 
[29] Freedman DS, Mei Z, Srinivasan SR, Berenson GS, Dietz WH. Cardiovascular risk factors and excess adiposity among overweight children and adolescents: the Bogalusa Heart Study. J Pediatr. 2007;150 (1):12-7 e2.

[30] Giapros VI, Challa AS, Cholevas VI, Evagelidou EN, Bairaktari ET, Andronikou SK. Vitamin D, parathormone, and insulin resistance in children born large for gestational age. J Pediatr Endocrinol Metab. 2014;27 (11-12):1145-50.

[31] Giese H, Konig LM, Taut D, Ollila H, Baban A, Absetz P, et al. Exploring the association between television advertising of healthy and unhealthy foods, self-control, and food intake in three European countries. Appl Psychol Health Well Being. 2015;7 (1):41-62.

[32] Goff DC, Jr., Lloyd-Jones DM, Bennett G, Coady S, D’Agostino RB, Sr., Gibbons R, et al. 2013 ACC/AHA guideline on the assessment of cardiovascular risk: a report of the American College of Cardiology/American Heart Association Task Force on Practice Guidelines. J Am Coll Cardiol. 2014;63 (25 Pt B):2935-59.

[33] Grigorakis DA, Georgoulis M, Psarra G, Tambalis KD, Panagiotakos DB, Sidossis LS. Prevalence and lifestyle determinants of central obesity in children. Eur J Nutr. 2016;55 (5):1923-31.

[34] Grosso G, Marventano S, Galvano F, Pajak A, Mistretta A. Factors associated with metabolic syndrome in a mediterranean population: role of caffeinated beverages. J Epidemiol. 2014;24 (4):327-33.

[35] Grosso G, Pajak A, Mistretta A, Marventano S, Raciti T, Buscemi S, et al. Protective role of the Mediterranean diet on several cardiovascular risk factors: evidence from Sicily, southern Italy. Nutr Metab Cardiovasc Dis. 2014;24 (4):370-7.

[36] Han JC, Lawlor DA, Kimm SY. Childhood obesity. Lancet. 2010;375 (9727):1737-48.

[37] Hardy LL, Bass SL, Booth ML. Changes in sedentary behavior among adolescent girls: a 2.5-year prospective cohort study. J Adolesc Health. 2007;40 (2):158-65.

[38] Hart CN, Carskadon MA, Considine RV, Fava JL, Lawton J, Raynor HA, et al. Changes in children's sleep duration on food intake, weight, and leptin. Pediatrics. 2013;132 (6):e1473-8o.

[39] Henderson M, Benedetti A, Barnett TA, Mathieu ME, Deladoey J, Gray-Donald K. Influence of Adiposity, Physical Activity, Fitness, and Screen Time on Insulin Dynamics Over 2 Years in Children. JAMA Pediatr. 2016;170 (3):227-35.

[40] Hitze B, Bosy-Westphal A, Bielfeldt F, Settler U, Plachta-Danielzik S, Pfeuffer M, et al. Determinants and impact of sleep duration in children and adolescents: data of the Kiel Obesity Prevention Study. Eur J Clin Nutr. 2009;63 (6):739-46. 
[41] Jaaskelainen P, Magnussen CG, Pahkala K, Mikkila V, Kahonen M, Sabin MA, et al. Childhood nutrition in predicting metabolic syndrome in adults: the cardiovascular risk in Young Finns Study. Diabetes Care. 2012;35 (9):1937-43.

[42] Javaheri S, Storfer-Isser A, Rosen CL, Redline S. Association of short and long sleep durations with insulin sensitivity in adolescents. J Pediatr. 2011;158 (4):617-23.

[43] Kanai Y, Kamoda T, Saito M, Fujiyama S, Nishimura K, Iwabuchi A, et al. Cord blood insulin-like growth factor (IGF)-1, IGF-binding proteins and adiponectin, and birth size in offspring of women with mild gestational diabetes. Early Hum Dev. 2016;93:39-42.

[44] Kang HT, Lee HR, Shim JY, Shin YH, Park BJ, Lee YJ. Association between screen time and metabolic syndrome in children and adolescents in Korea: the 2005 Korean National Health and Nutrition Examination Survey. Diabetes Res Clin Pract. 2010;89 (1):72-8.

[45] Klingenberg L, Chaput JP, Holmback U, Visby T, Jennum P, Nikolic M, et al. Acute Sleep Restriction Reduces Insulin Sensitivity in Adolescent Boys. Sleep. 2013;36 (7):108590.

[46] Koren D, Levitt Katz LE, Brar PC, Gallagher PR, Berkowitz RI, Brooks LJ. Sleep architecture and glucose and insulin homeostasis in obese adolescents. Diabetes Care. 2011;34 (11):2442-7.

[47] Koskinen J, Magnussen CG, Sabin MA, Kahonen M, Hutri-Kahonen N, Laitinen T, et al. Youth overweight and metabolic disturbances in predicting carotid intimamedia thickness, type 2 diabetes, and metabolic syndrome in adulthood: the Cardiovascular Risk in Young Finns study. Diabetes Care. 2014;37 (7):1870-7.

[48] Kris-Etherton P, Eckel RH, Howard BV, St Jeor S, Bazzarre TL, Nutrition Committee Population Science C, et al. AHA Science Advisory: Lyon Diet Heart Study. Benefits of a Mediterranean-style, National Cholesterol Education Program/American Heart Association Step I Dietary Pattern on Cardiovascular Disease. Circulation. 2001;103 (13):1823-5.

[49] Li S, Chen W, Srinivasan SR, Xu J, Berenson GS. Relation of childhood obesity/cardiometabolic phenotypes to adult cardiometabolic profile: the Bogalusa Heart Study. Am J Epidemiol. 2012;176 Suppl 7:S142-9.

[50] Loy J, Youn HA, Schwack B, Kurian M, Ren Fielding C, Fielding GA. Improvement in nonalcoholic fatty liver disease and metabolic syndrome in adolescents undergoing bariatric surgery. Surg Obes Relat Dis. 2015;11 (2):442-9.

[51] Ludwig DS, Peterson KE, Gortmaker SL. Relation between consumption of sugarsweetened drinks and childhood obesity: a prospective, observational analysis. Lancet. 2001;357 (9255):505-8. 
[52] Maddison R, Foley L, Ni Mhurchu C, Jiang Y, Jull A, Prapavessis $H$, et al. Effects of active video games on body composition: a randomized controlled trial. Am J Clin Nutr. 2011;94 (1):156-63.

[53] Marcil V, Amre D, Seidman EG, Boudreau F, Gendron FP, Menard D, et al. Hepatocyte nuclear factor 4 alpha polymorphisms and the metabolic syndrome in FrenchCanadian youth. PLoS One. 2015;10 (2):e0117238.

[54] Mark AE, Janssen I. Relationship between screen time and metabolic syndrome in adolescents. J Public Health (0xf). 2008;30 (2):153-60.

[55] Matthews KA, DahI RE, Owens JF, Lee L, Hall M. Sleep duration and insulin resistance in healthy black and white adolescents. Sleep. 2012;35 (10):1353-8.

[56] Matthews VL, Wien $M$, Sabate J. The risk of child and adolescent overweight is related to types of food consumed. Nutr J. 2011;10:71.

[57] Monzani A, Rapa A, Fuiano N, Diddi G, Prodam F, Bellone S, et al. Metabolic syndrome is strictly associated with parental obesity beginning from childhood. Clin Endocrinol (Oxf). 2014;81 (1):45-51.

[58] Muller UM, Walther C, Adams V, Mende M, Adam J, Fikenzer K, et al. Long term impact of one daily unit of physical exercise at school on cardiovascular risk factors in school children. Eur J Prev Cardiol. 2016;23 (13):1444-52.

[59] Nakashima M, Sakurai M, Nakamura K, Miura K, Yoshita K, Morikawa Y, et al. Dietary glycemic index, glycemic load and blood lipid levels in middle-aged Japanese men and women. J Atheroscler Thromb. 2010;17 (10):1082-95.

[6o] Nanri A, Mizoue T, Noda M, Takahashi Y, Kato $M$, Inoue $M$, et al. Rice intake and type 2 diabetes in Japanese men and women: the Japan Public Health Center-based Prospective Study. Am J Clin Nutr. 2010;92 (6):1468-77.

[61] Nupponen M, Pahkala K, Juonala M, Magnussen CG, Niinikoski H, Ronnemaa T, et al. Metabolic syndrome from adolescence to early adulthood: effect of infancyonset dietary counseling of low saturated fat: the Special Turku Coronary Risk Factor Intervention Project (STRIP). Circulation. 2015;131 (7):605-13.

[62] O'Neil CE, Fulgoni VL, 3rd, Nicklas TA. Tree Nut consumption is associated with better adiposity measures and cardiovascular and metabolic syndrome health risk factors in U.S. Adults: NHANES 2005-2010. Nutr J. 2015;14:64.

[63] Park SH, Lee KS, Park HY. Dietary carbohydrate intake is associated with cardiovascular disease risk in Korean: analysis of the third Korea National Health and Nutrition Examination Survey (KNHANES III). Int J Cardiol. 2010;139 (3):234-40.

[64] Perng W, Gillman MW, Mantzoros CS, Oken E. A prospective study of maternal prenatal weight and offspring cardiometabolic health in midchildhood. Ann Epidemiol. 2014;24 (11):793-800 e1. 
[65] Pontiroli AE. Type 2 diabetes mellitus is becoming the most common type of diabetes in school children. Acta Diabetol. 2004;41 (3):85-90.

[66] Ramirez-Silva I, Rivera JA, Trejo-Valdivia B, Martorell R, Stein AD, Romieu I, et al. Breastfeeding status at age 3 months is associated with adiposity and cardiometabolic markers at age 4 years in Mexican children. J Nutr. 2015;145 (6):1295-302.

[67] Razquin C, Martinez JA, Martinez-Gonzalez MA, Mitjavila MT, Estruch R, Marti A. A 3 years follow-up of a Mediterranean diet rich in virgin olive oil is associated with high plasma antioxidant capacity and reduced body weight gain. Eur J Clin Nutr. 2009;63 (12):1387-93.

[68] Redline S, Storfer-Isser A, Rosen CL, Johnson NL, Kirchner HL, Emancipator J, et al. Association between metabolic syndrome and sleep-disordered breathing in adolescents. Am J Respir Crit Care Med. 2007;176 (4):401-8.

[69] Sabate J, Wien M. A perspective on vegetarian dietary patterns and risk of metabolic syndrome. Br J Nutr. 2015;113 Suppl 2:S136-43.

[70] Shamsuzzaman A, Szczesniak RD, Fenchel MC, Amin RS. Glucose, insulin, and insulin resistance in normal-weight, overweight and obese children with obstructive sleep apnea. Obes Res Clin Pract. 2014;8 (6):e584-91.

[71] Skouteris H, McCabe M, Swinburn B, Newgreen V, Sacher P, Chadwick P. Parental influence and obesity prevention in pre-schoolers: a systematic review of interventions. Obes Rev. 2011;12 (5):315-28.

[72] Song S, Young Paik H, Song WO, Song Y. Metabolic syndrome risk factors are associated with white rice intake in Korean adolescent girls and boys. Br J Nutr. 2015;113 (3):479-87.

[73] Spruyt K, Molfese DL, Gozal D. Sleep duration, sleep regularity, body weight, and metabolic homeostasis in school-aged children. Pediatrics. 2011;127 (2):e345-52.

[74] Stoger R. Epigenetics and obesity. Pharmacogenomics. 2008;9 (12):1851-60.

[75] Trost SG, Sundal D, Foster GD, Lent MR, Vojta D. Effects of a pediatric weight management program with and without active video games a randomized trial. JAMA Pediatr. 2014;168 (5):407-13.

[76] Velazquez-Lopez L, Santiago-Diaz G, Nava-Hernandez J, Munoz-Torres AV, MedinaBravo P, Torres-Tamayo M. Mediterranean-style diet reduces metabolic syndrome components in obese children and adolescents with obesity. BMC Pediatr. 2014; $14: 175$.

[77] White MJ, Eren F, Agirbasli D, Williams SM, Agirbasli M. SHBG gene polymorphism (rs1799941) associates with metabolic syndrome in children and adolescents. PLoS One. 2015;10 (2):e0116915. 
[78] Xu S, Xue Y. Pediatric obesity: Causes, symptoms, prevention and treatment. Exp Ther Med. 2016;11 (1):15-20.

[79] Yang J, Farioli A, Korre M, Kales SN. Modified Mediterranean diet score and cardiovascular risk in a North American working population. PLoS One. 2014;9 (2):e87539.

[80] Zeisel SH. Epigenetic mechanisms for nutrition determinants of later health outcomes. Am J Clin Nutr. 2009;89 (5):1488S-93S.

[81] Zhang H, Zhang T, Li S, Li Y, Hussain A, Fernandez C, et al. Long-term Impact of Childhood Adiposity on Adult Metabolic Syndrome Is Modified by Insulin Resistance: The Bogalusa Heart Study. Sci Rep. 2015;5:17885.

[82] Zhu Y, Li AM, Au CT, Kong AP, Zhang J, Wong CK, et al. Association between sleep architecture and glucose tolerance in children and adolescents. J Diabetes. 2015;7 (1):10-5. 